\title{
A Coupled, Multiphase Heat Flux Boundary Condition for the Discrete Element Method
}

\author{
Aaron M. Lattanzi, Christine M. Hrenya* \\ University of Colorado at Boulder, Dept. of Chemical and Biological Engineering, Boulder, CO, USA \\ *corresponding author
}

\begin{abstract}
Similar to single-phase flows, multiphase systems with a constant heat flux at the wall are common in practice. Unlike single-phase flows, however, the numerical implementation of a constant boundary flux is non-trivial due to the coupling between phases - i.e., the partition of total flux between the phases can vary in space and time. A numerical technique for modeling such a boundary condition is proposed here and verified via simulations of gas-solid flows. While discrete-particle simulations of monodisperse particles are considered here, the technique can be extended to include radiation, polydisperse systems, and/or a continuum representation of the solids phase.
\end{abstract}

\section{Introduction}

The transfer of thermal energy in a gas-solid mixture is critical to many industrial processes, with modern examples including, but not limited to: rotary kilns [5, 9, 22], fluidized beds $[12,17,18,2731]$, packed beds [6, 29, 30], and heat exchangers with granular media as the heat transfer fluid [13]. Products resulting from the aforementioned applications vary widely, and encompass commodities such as limestone, petroleum, polymers, and pharmaceuticals. Despite the pervasiveness of multiphase heat transfer, a fundamental description of the underlying physics still requires further development.

Approaches to modeling heat transfer in a gas-solid flow can be classified by their treatment of the solids phase. The discrete element method (DEM) [7, 8, 19] involves the tracking of individual particles, while a two-fluid model (TFM) treats the solid phase as a continuum. For both approaches, the vast majority of previous modeling efforts have been limited to constant-temperature boundary conditions $[5,9,12,13,14,15,18,22,27]$. However, the constant heat flux boundary condition is of considerable practical importance. For example, unit operations involving heat generation from combustion, reactions, and/or solar heating, are more appropriately described by a specified flux at the boundary than a constant temperature. Moreover, the temperature gradients within the wall play an integral role in the evaluation of mechanical stresses and/or selection of construction materials for the given operation. Unfortunately, the representation of a constant flux boundary condition presents challenges for both DEM and TFM frameworks. Specifically, a constant total heat flux is currently achieved by imposing a constant heat flux upon each phase. In practice, however, the total heat flux to the mixture typically remains constant, while the flux to each phase varies in space and/or time. Few attempts to quantify the latter interpretation of a constant heat flux boundary condition have been undertaken $[1,2]$, and none have been extended to multiphase flows described using the DEM or TFM framework.

In the current work, we propose a new method for imposing a total heat flux boundary condition on multiphase flows. The new boundary condition (BC) is based on an assumption that all phases experience the same local wall temperature and that heat transfer to each phase occurs in parallel. The resulting BC couples the heat flux at the wall for the gas and solid phases, 
and also allows the heat fluxes to each phase to vary with space and/or time. The general methodology can be adapted for use in discrete-particle or continuum-solid frameworks, but the focus here is on gas-solid flows simulated using computational fluid dynamics - discrete element method (CFD-DEM). Various systems with a specified heat flux imposed at the boundary were considered. The accuracy of the new BC was verified by comparing the wall heat flux obtained from simulations with the imposed values. Moreover, the wall heat fluxes of each phase are shown to vary in space and time, while still maintaining the total specified flux - i.e., the heat fluxes of each phase are appropriately coupled.

\section{Computational Methodology}

All simulations were performed via MFIX [24, 28], an open-source software developed by the U. S. National Energy Technology Laboratory (NETL) for multiphase flows. Specifically, the hydrodynamics and heat transfer of a gas-solids flow were simulated using the computational fluid dynamics and discrete element method (CFD-DEM) framework. The CFD portion treats the fluid phase as a continuum and employs a numerical grid that is much larger than the diameter of a particle. Particles are treated as discrete entities via DEM. Coupling of the continuum and discrete phases in CFD-DEM occurs via interphase transfer of momentum (drag) and heat (convection). The corresponding governing equations are outlined below.

\section{Fluid Phase}

The gas phase is treated as a continuum via the following continuity and momentum balances, respectively [3]:

$\partial \partial \mathrm{t} \varepsilon g \rho g+\nabla \cdot \varepsilon g \rho g \mathbf{v g}=0$

$\partial \partial \mathrm{t} \varepsilon g \rho g \mathbf{v g}+\nabla \cdot \varepsilon g \rho g \mathbf{v g v g}=-\varepsilon g \nabla P g+\nabla \cdot \tau \mathbf{g}-i=1 n \beta g, s, i \mathbf{v g}-\mathbf{v p}, \mathbf{i}+\varepsilon g \rho g \mathbf{g}$

$\beta g, s, i 1501-\varepsilon g 2 \mu g \varepsilon g d p, i+741-\varepsilon g \rho g \mathbf{v g}-\mathbf{v p}, \mathbf{i} d p, i \quad \varepsilon g<0.8$

$34 C D \varepsilon g 1-\varepsilon g \rho g \mathbf{v g}-\mathbf{v p}, \mathbf{i} d p, i \varepsilon g-2.65 \quad \varepsilon g \geq 0.8$

where $\varepsilon g$ is the gas fraction, $\rho g$ is the gas density, vgis the gas velocity, $P g$ is the gas pressure, $\tau \mathbf{g}=\varepsilon g \mu g \nabla \mathbf{v g}+\nabla \mathbf{v g T}+\varepsilon g \lambda g \operatorname{tr} 1 / 2 \nabla \mathbf{v g}+\nabla \mathbf{v g T}$ is the gas-phase stress, $\mu g$ is the gas dynlähic viscosity, $\lambda g$ is the gas bulk viscosity, $\beta g, s, i$ is the interphase momentum transfer coefficient for particle $i[10], \mathbf{v p , i}$ is the velocity of particle $i, n$ is the number of particles within the CFD cell, $d p, i$ is the diameter of particle $i, C D$ is the coefficient of drag, and $\mathbf{g}$ is the gravity vector. The balance for the internal (thermal) energy of the gas is treated in a similar manner:

$\partial \partial \mathrm{t} \varepsilon g \rho g C p, g T g+\nabla \cdot \varepsilon g \rho g C p, g T g \mathbf{v g}=-\nabla \cdot \mathbf{q} " \mathbf{g}-i=1 n h g, s, i T g-T p, i$

q" $\mathbf{g}=-\varepsilon g k g \nabla T g$

where $C p, g$ is the gas specific heat, $T g$ is the (thermal) gas temperature, $\mathbf{q}^{\prime \prime} \mathbf{g}$ is the gas heat $\left(\mathrm{fF}_{\mathrm{Ix}}\right.$, $k g$ is the gas thermal conductivity, $h g, s, i$ is the interphase heat transfer coefficient for particle $i$, and $T p, i$ is the temperature of particle $i$.

Solid Phase 
In contrast to the continuum representation of the gas phase, the solid phase is treated as discrete particles via DEM. Particles are modeled using a soft-sphere approach, and thus can undergo multiple, enduring contacts. The time evolution of each particle property takes the form of an initial value problem. Since the time step for numerical integration must be smaller than the duration of a collision [25], the DEM time step is generally orders of magnitude smaller than the continuum time step; i.e., the DEM solver completes many time steps per CFD solver time step.

The force balance resulting from the application of Newton's second law to each particle governs particle motion with time:

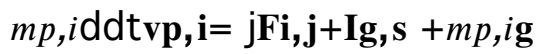

$\mathbf{I g}, \mathbf{s}=-V p, i \nabla P g+V p, i \beta g, s, i \varepsilon s \mathbf{v g}-\mathbf{v p}, \mathbf{i}$

where $m p, i=\rho p, i V p, i$ is the mass of particle $i, \rho p, i$ is the density of particle $i, V p, i$ is the volume of particle $i, \mathbf{F i}, \mathbf{j}$ is the force resulting from contacts between particle $i$ and solid-body $j$ (particle or wall), and Ig,s is the drag force. The linear-spring-dashpot method is used to describe particle contacts:

$$
\begin{gathered}
\mathbf{F i}, \mathbf{j}=-\kappa n \boldsymbol{\sigma}-\eta n \gamma n, c r i t \mathrm{~d} \boldsymbol{\sigma} \mathrm{d} t \\
e=\exp -\pi \eta n 1-\eta n 2 \quad \gamma n, c r i t=2 m e f f \kappa n
\end{gathered}
$$

where $e$ is the restitution coefficient (0.90), $\kappa n$ is the spring constant $(2000 \mathrm{~N} / \mathrm{m}), \gamma n, c r i t$ is the critical damping coefficient (recovered in the limit of perfectly inelastic collisions $e=0$ ), $\eta n$ is the ratio of the damping coefficient to the critical damping coefficient, and $\sigma$ is the "overlap" of particle $i$ and body $j$. It should be noted that an artificially soft spring constant is used in this work in order to increase the computational efficiency of the DEM framework. However, artificially soft spring constants have been shown to yield artificially high heat transfer, due to increased contact times and overlap [15]. A means of correcting for the softened spring constant is also given in [15], referred to here as "area" and "time correction", though such corrections are not employed in this work for simplicity. Similarly, friction is neglected in this work, and thus no governing equation for the angular momentum is required. It is important to note that although the inclusion of area and time corrections as well as friction will affect the solids hydrodynamics and heat transfer, but does not impact the implementation or accuracy of the boundary condition developed herein. The qualitative nature of these effects will be discussed further in Results.

With regard to heat transfer, radiation is not considered here and thus conduction and convection are the only relevant heat transfer mechanisms. The conductive mechanisms may be divided into two categories: (1) direct conduction stemming from the contact of two solids (PP, $\mathrm{PW}$ ) and (2) indirect conduction through the thin layer of fluid between two solids (PFP, PFW). Accordingly, the mechanisms under consideration are: particle-particle (PP) conduction, particlefluid-particle (PFP) conduction, particle-wall (PW) conduction, particle-fluid-wall (PFW) conduction, and interphase convection. The resulting internal energy balance of a particle is [14]:

$m p, i C p, i \partial \partial \mathrm{t} T p, i=\mathrm{j} q i, j P P+q i, j P F P+q i P W+q i P F W+h g, s, i T g-T p, i$ 
where $C p, i$ is the specific heat of particle $i, q i, j$ is the rate of heat transfer between particle $i$ and particle $j$, and $q i$ is the rate of heat transfer between particle $i$ and a wall. Note that an inherent assumption of Eq. (9) is an isothermal particle or Biot number $<<1$ (i.e., no spatial temperature gradients within a particle); the methods developed here can also be adapted to non-isothermal particles as developed by [16].

Since the aim of this work is directed at a new boundary condition for use in CFD-DEM simulations, only the mechanisms pertaining to heat transfer with a wall ( $q i P W$ and $q i P F W$ ) will be expounded upon here. Further details on direct conduction [4], indirect conduction [21], and convection $[11,20]$ are available elsewhere, and the closures used for $(q i, j P P, q i, j P F P$, and $h g, s, i)$ in this work are identical to those used by [14]. The particle-wall (direct) conduction takes the form $[4,14]$ :

$$
q i P W=h p w, i T w-T p, i=4 k p, i k w k p, i+k w R C T w-T p, i
$$

where $h p w, i$ is the particle-wall conduction heat transfer coefficient for particle $i, k p, i$ is the thermal conductivity of particle $i, k w$ is the thermal conductivity of the wall, $T w$ is the wall temperature, and $R C=d p, i \sigma-\sigma 2$ is the radius of contact (resulting from "overlap" of the particle and wall upon collision).

For particle-fluid-wall (indirect) conduction, an adaptation of the static fluid lens model [21] is utilized [14]; see Figure 1. The underlying principle of the model is that each particle is surrounded by a static fluid lens, the thickness of which is a fraction of the particle diameter. When this lens (rather than the particle itself) overlaps with a solid body (wall or other particles), one-dimensional conduction through the fluid lens is assumed to occur between the particle and solid body.

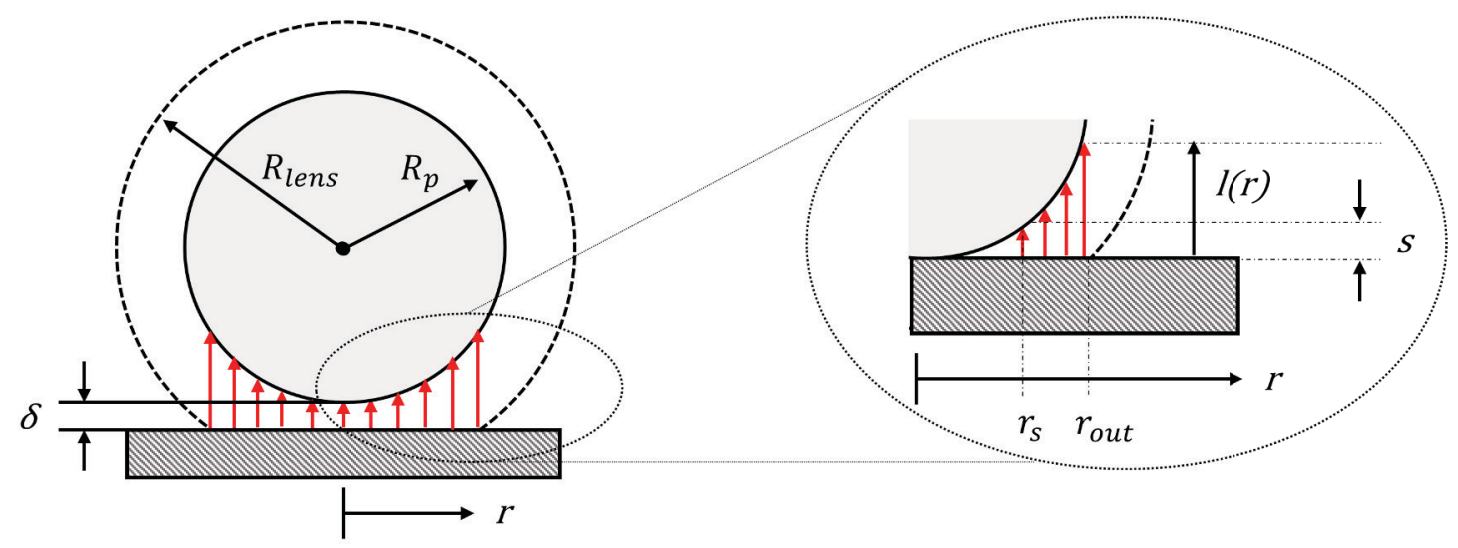

Figure 1. Illustration of particle-fluid-wall conduction via static-fluid lens (dashed

In mathematical terms, the closure for particle-fluid-wall conduction takes the form:

$q i P F W=h p f w, i T w-T p, i=$ rinrout $2 \pi K g r M a x(l, s) T w-T p, i d r$

rin $=r s=R p 2-s-R p-\delta 2 \delta \leq s 0 \delta>s$

rout $=$ Rlens $2-R p+\delta 2 \delta>$ Rlens $2-R p 2-R p R p \delta \leq R l e n s 2-R p 2-R p$ 
where $h p f w, i$ is the particle-fluid-wall conduction heat transfer coefficient for particle $i$. The lower integration bound (rin) in Eq. 11 depends on whether the bottom of the particle is within the minimum conduction distance $(s)$ of the wall. For the case of a particle-wall separation distance $(\delta)$ greater than the minimum conduction distance $(\delta>s)$, the lower bound of integration is zero. For the opposite case $(\delta \leq s)$, the lower integration bound becomes the radial position $\left(r_{s}\right)$ at which the conduction distance $(l)$ equals the minimum conduction distance $(s)$. A minimum conduction distance is needed in two cases. First, the presence of surface asperities leads to finite separation between the particle and wall. Second, rarefaction effects become non-negligible as the separation distance decreases to the order of magnitude of the mean free path of the gas. These physical justifications for a minimum separation distance also have a mathematical counterpart; namely, a singularity will develop in the integrand of Eq. 11 as the conduction distance ( $l$ ) approaches zero - i.e., solid-body contact. Since the particles are assumed to be perfectly smooth, a minimum conduction distance on the order of magnitude of the mean free path of the gas is employed here $(s=2.75 \mathrm{E}-8 \mathrm{~m})$. The upper integration bound (rout) is the intersection point between the fluid lens and wall, and is constrained to be less than the particle radius $(R p)$. The thickness of the fluid lens can have a noticeable impact on the solids heat transfer, but does not affect the implementation of the boundary condition. For this reason, the fluid lens thickness utilized in other works [14,29] was adopted here and kept constant $($ Rlens $=1.4 R p)$.

\section{Development of Heat Flux Boundary Condition}

When considering heat transfer with a boundary, three classical conditions arise: (1) constant wall temperature (Dirichlet), (2) constant wall flux (Neumann), or (3) a linear combination of types $1 \& 2$ (Robin or mixed). For single-phase systems, the implementation of a constant wall flux (Neumann) boundary condition (BC) into a CFD code is straightforward and well documented. However, the state-of-the-art for extending a Neumann BC to multiphase flows is to specify a constant heat flux to each phase [26]. Practically speaking, it is often the total heat flux to the mixture that remains constant, while the fluxes to each phase may vary in space and time. For this more general case of constant total flux to the mixture, we assume here that (i) both the gas and solid phases experience the same wall temperature and (ii) the heat transfer to each phase occurs in parallel. Therefore, the total heat flux at the wall can be written as a sum of the heat fluxes to each phase, which may vary with time and space:

$q^{\prime \prime} w=q^{\prime \prime} g(\mathbf{x}, t)+q " s(\mathbf{x}, t)$

Since Eq. 12 couples the gas and solid frameworks (CFD and DEM) at the boundary, special attention is required to close the heat flux terms. More specifically, because the gas phase is treated as a continuum, the solution of its internal energy balance yields a single temperature for a given numerical (CFD) cell, including the cell adjacent to the wall. Correspondingly, the wall temperature used for the particle heat transfer (Eq. 10-11) is dictated by the CFD cell it resides in (i.e., wall temperature gradients smaller than the CFD cell that may arise from the discrete nature of the particles cannot be resolved within a CFD cell). Therefore, the heat flux to 
the gas phase and solid phase must be given on a CFD-grid basis. The gas-phase flux $q$ " $g$ is already in terms of continuum variables (Eq. 5). However, the heat flux to each particle is resolved in DEM (Eq. 10-11) and thus $q$ "s is not in terms of a CFD-grid basis a priori. To recast the individual particle fluxes obtained via DEM for implementation in a CFD cell (continuum), the solids flux is taken as the spatial and temporal average of the total particle-wall conduction occurring within a given CFD cell and over a continuum time step:

$q^{\prime \prime} s(\mathbf{x}, t)=1 p A c j=1 p i=1 n j H s, i T w-T p, i=T w A-B$

$A=j=1 p i=1 n j H s, i p A c \quad B=j=1 p i=1 n j H s, i p A c T p, i \quad H s, i=h p w, i+h p f w, i$

where $p$ is the number of DEM time steps within a continuum time step, $A c$ is the area of the CFD cell adjacent to the wall, $n j$ is the number of particles within a CFD cell adjacent to the wall during the $j^{\text {th }}$ DEM time step, $T w$ is the continuum wall temperature, and $H s, i$ is the total particle-wall heat transfer coefficient for particle $i$. Since the continuum and DEM frameworks are coupled explicitly (i.e., values at the current time step depend on previous time step only), the (continuum) wall temperature $T_{w}$ in Eq. 13 will be constant over all of the DEM iterations associated with a single fluid time step. Substitution of the solids heat flux (Eq. 13) and the gas heat flux (Eq. 5) for a boundary cell into Eq. 12 gives a closed form for the wall flux in a given cell on a CFD (continuum) basis. Spatial discretization of said equation yields the following relations:

$q^{\prime \prime} w=\varepsilon g, k-1 t k g, k-1 t T g, k t+1-T g, k-1 t+1 d x k+T g, k-1 / 2 t+1 A t-B t$

$T g, k t+1-T g, k-1 t+1 d x k+A t \varepsilon g, k-1 t k g, k-1 t T g, k-1 / 2 t+1=q^{\prime \prime} w+B t \varepsilon g, k-1 t k g, k-1 t$

$\partial \operatorname{Tg} \partial n+H w(\mathbf{x}, t) \operatorname{Tg}=C(\mathbf{x}, t)$

where $n$ is the outward normal to the boundary, $k$ refers to the spatial coordinate, $t+1$ is the current time step, $t$ is the previous time step, $H w=A t / \varepsilon g, k-1 t k g,(16-1 t$, $C=Q " w+B t / \varepsilon g, k-1 t k g, k-1 t$ and $T g, k-1 / 2 t+1=1 / 2 T g, k t+1+T g, k-1 t+1$. Inspection of Eq. 15 and 16 shows that the constant flux BC for the mixture is now formulated as a Robin BC. By contrast, if radiative mechanisms are accounted for, the boundary condition is more naturally formulated as a Dirichlet boundary condition, which depends upon space and time. However, if this approach is taken, the desired root of a fourth order polynomial must be found for each CFD cell at each continuum time step.

The above methodology for implementing a constant wall flux into the CFD-DEM framework can be completed for any number of phases and easily extended to the two-fluid model (solids and gas both treated as a continuum). Finally, since this method involves a distribution of the total wall flux between $M$ phases, the dependence upon other phase temperatures (through the B term(s) in Eq. 15) can be handled explicitly and there is no need for nonstandard matrices or preconditioning (like in the case of interphase heat transfer) $[23,26]$.

\section{Systems Simulated}


In this work, the thermodynamic properties for the gas phase were treated as functions of thermal temperature while the solids properties were treated as constants. Inputs used for the gas and solid phases are outlined in Table 1 and 2, respectively.

Table 1: Gas properties

\begin{tabular}{llll}
\hline \multicolumn{2}{l}{ Species: Air } & & \\
\hline$\rho g$ & $\rho g=P g M w R T g$ & & \\
$k g$ & $k g=k g, r e f T g T g, r e f$ & $k g, r e f=0.0252 \mathrm{Wm} \mathrm{K}$ & $T g, r e f=300 \mathrm{~K}$ \\
$C p, g$ & $\begin{array}{l}1004.2 \mathrm{Jkg} \mathrm{K} \\
\mu g=\mu g, r e f T g T g, r e f 32 T g, r e f+s T g+\end{array}$ & & \\
$\mu g$ & $s$ & $\mathrm{~S}=110.4 \mathrm{~K}$ & $T g, r e f=300 \mathrm{~K}$ \\
& $\mu g, r e f=1.72 * 10-5 \quad P a s$ & & \\
\hline
\end{tabular}

Table 2: Solid properties

Species: Sand $\left(\mathrm{SiO}_{2}\right)$

$\rho p, i \quad 2582 \mathrm{kgm} 3$

$k p, i \quad 1.402 \mathrm{Wm} \mathrm{K}$

Cp,i $794.96 \mathrm{Jkg} \mathrm{K}$

$d p, i \quad 200 \mu m$

A total of five different geometries were simulated with the new BC outlined above, as illustrated in Figure 2. The first four geometries are falling-particle, vertical heat exchangers with internal baffles. A constant mass inflow of gas and solids was imposed at the top of the heat exchangers and a constant, positive heat flux at the wall (into domain) was imposed upon all side walls. The vertical heat exchangers exhibit two distinct properties that impact the developed BC. First, a transient period exists where the initially empty domain experiences an increase in particle concentration from the mass inflow. The solids heat flux for each CFD cell will be zero until particles enter the cell and undergo conduction with the wall. If the boundary flux is large during this transient period, the solids heat flux will exhibit large gradients in space (like at the leading edge of the solids flow) and time, leading to numerical instabilities; to mitigate this, the wall heat flux was ramped linearly from 0 to $4200 \mathrm{~W} / \mathrm{m} 2$ over a time of $0.5 \mathrm{~s}$. Second, the system eventually reaches a statistical steady state. Explicit phase coupling by CFD-DEM 
requires that the " $\mathrm{A}$ " and " $\mathrm{B}$ " terms in the $\mathrm{BC}$ (Eq. 15) come from the previous DEM iteration. A physical interpretation of the explicit coupling is that the solids heat flux at the current time step is approximated by that at the previous time step; at steady state, this approximation should be sufficiently accurate. The fifth simulation geometry, however, is a bubbling-bed heat exchanger with a constant positive wall flux imposed upon all side walls. In contrast to the falling-particle heat exchangers, the bubbling bed does not reach a stable steady state due to the presence of bubbling instabilities. Accordingly, the solids-phase heat flux at the wall may change dramatically in space and/or time, thereby providing a more stringent test of the explicit approximation used to formulate the new BC. The numerical and geometric inputs for all of the cases are given in Table 3 and 4.

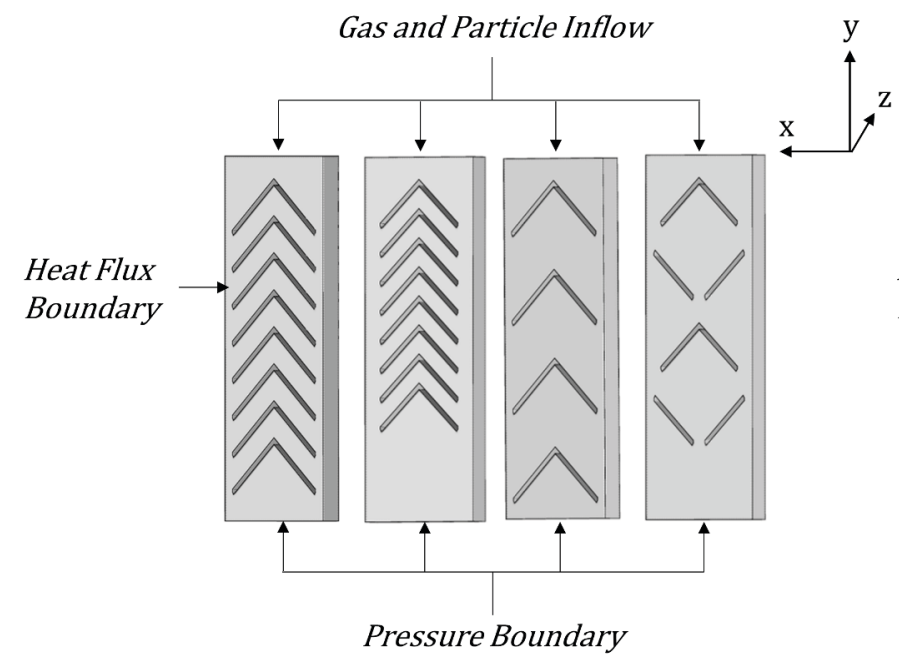

(a)

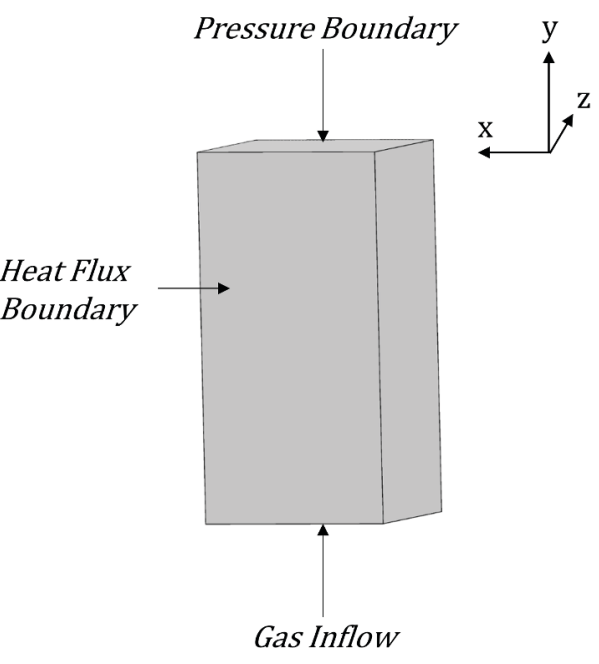

Gas Inflow

Figure 2. Schematics of (a) vertical, falling-particle heat exchangers and (b) huthblina hed heat exrhancer

Table 3: Simulation inputs: Geometry and Operating Conditions

\begin{tabular}{lll}
\hline $\begin{array}{l}\text { Configurations } 1-4 \\
\text { Column Height }\end{array}$ & $9 \mathrm{~cm}$ & \\
Column Width & $3 \mathrm{~cm}$ & \\
Column Depth & $0.65 \mathrm{~cm}$ & \\
Number of Cells & $138 \times 46 \times 10$ \\
Mass Flux & $180 \mathrm{kgm} 2 \mathrm{~s}$ & $\varepsilon s=0.50$
\end{tabular}




\begin{tabular}{ll} 
Tg/s,inlet & $300 \mathrm{~K}$ \\
Pg,outlet & $101.325 \mathrm{kPa}$ \\
Qwall & $4200 \mathrm{Wm} 2$ \\
& \\
Configuration 5 & \\
Column Height & $2 \mathrm{~cm}$ \\
Column Width & $1 \mathrm{~cm}$ \\
Column Depth & $1 \mathrm{~cm}$ \\
Number of Cells & $100 \times 50 \times 50$ \\
Vg,inlet & $35 \mathrm{cms}$ \\
Tg,inlet & $300 \mathrm{~K}$ \\
Tg/s,initial & $300 \mathrm{~K}$ \\
Pg,outlet & $101.325 \mathrm{kPa}$ \\
Qwall & $4200 \mathrm{Wm} 2$ \\
\hline
\end{tabular}

Table 4: Dimensions for Falling-Particle Heat Exchanger

\begin{tabular}{ll}
\hline Configuration 1 & \\
Baffle-Wall Spacing & $0.228 \mathrm{~cm}$ \\
Baffle-Baffle Spacing & $-0.521 \mathrm{~cm}$ \\
Baffle Angle & $45 \mathrm{Deg}$ \\
& \\
Configuration 2 & \\
Baffle-Wall Spacing & $0.424 \mathrm{~cm}$
\end{tabular}




\begin{tabular}{ll} 
Baffle-Baffle Spacing & $-0.521 \mathrm{~cm}$ \\
Baffle Angle & $45 \mathrm{Deg}$ \\
& \\
Configuration 3 & \\
Baffle-Wall Spacing & $0.228 \mathrm{~cm}$ \\
Baffle-Baffle Spacing & $0.783 \mathrm{~cm}$ \\
Baffle Angle & $45 \mathrm{Deg}$ \\
& \\
Configuration 4 & \\
Baffle-Wall Spacing & $0.424 \mathrm{~cm}$ \\
Baffle-Baffle Spacing & $0.521 \mathrm{~cm}$ \\
Baffle Angle & $45 \mathrm{Deg}$ \\
Hopper-Wall Spacing & $0.228 \mathrm{~cm}$ \\
Hopper-Hopper Gap & $0.326 \mathrm{~cm}$ \\
Hopper Angle & $45 \mathrm{Deg}$ \\
\hline
\end{tabular}

\section{Results}

To validate the accuracy of the new total flux BC for CFD-DEM, all five configurations were simulated with a prescribed positive (inward) heat flux at the wall. Simulations of the falling-particle heat exchanger configurations, which reach a statistical steady state, are considered first. In Figure 3, the resulting gas volume fraction (top row) and wall temperature (bottom row) profiles demonstrate that regions of lower gas volume fraction (higher solids concentration) result in lower wall temperatures, consistent with expectations. In particular, the solids heat transfer coefficient is much larger than the gas heat transfer coefficient when a high number of particle-wall contacts occur, and that particles in this work have the ability to store significantly more energy than the fluid $(\rho p, i C p, i / \rho g C g 1)$, so regions of high solids fraction result in cooler walls.

For a more detailed view of the temperature profiles, the data along the right side walls was extracted and plotted in Figure 4. The predicted wall temperatures lead to a "hot spot" near the inlet (at large column heights) for all four configurations, which can be attributed to the rapid dilution of solids as they undergo gravitational acceleration prior to the first baffle. Depending upon the baffle-wall spacing, particle buildup will occur at or above the first baffle and correspond to a decrease in wall temperature due to high heat transfer to the solid phase. After the first baffle, the particles again accelerate and generally become more dilute, leading to slight increases in wall temperature down the length of the column. Furthermore, the alternating baffle configuration (far right) yields wall temperatures that are substantially higher than any other baffle configuration, and thus it is the least desirable geometry for maximizing heat transfer.

Since the conduction mechanisms for the solids phase ( $q i P W, q i P F W$ ) are primarily a function of particle-wall proximity, they are strongly influenced by system hydrodynamics. As noted above, the softened spring constant utilized here will lead to enhanced solids heat transfer. Area and time correction have recently been developed such that artificially-soft particles can 
still be used to simulate stiffer materials while maintaining physical accuracy [15]. Inclusion of such corrections will reduce the level of solids-phase conduction. The reduction in solids heat transfer will ultimately lead to hotter wall temperatures. By contrast, the inclusion of angular momentum would work in direct opposition to area and time correction. More specifically, angular momentum would serve to further dissipate energy during particle-wall collisions, due to frictional contacts now being accounted for. Particle-wall contacts will be more pronounced in the more dissipative system and thus serve to increase heat transfer, resulting in cooler wall temperatures. It is worth noting that the artificially-soft particles (without area and time corrections) and lack of friction simulated here was done for purposes of simplicity only, and such simplifications do not impact the implementation or validation of the new boundary condition.
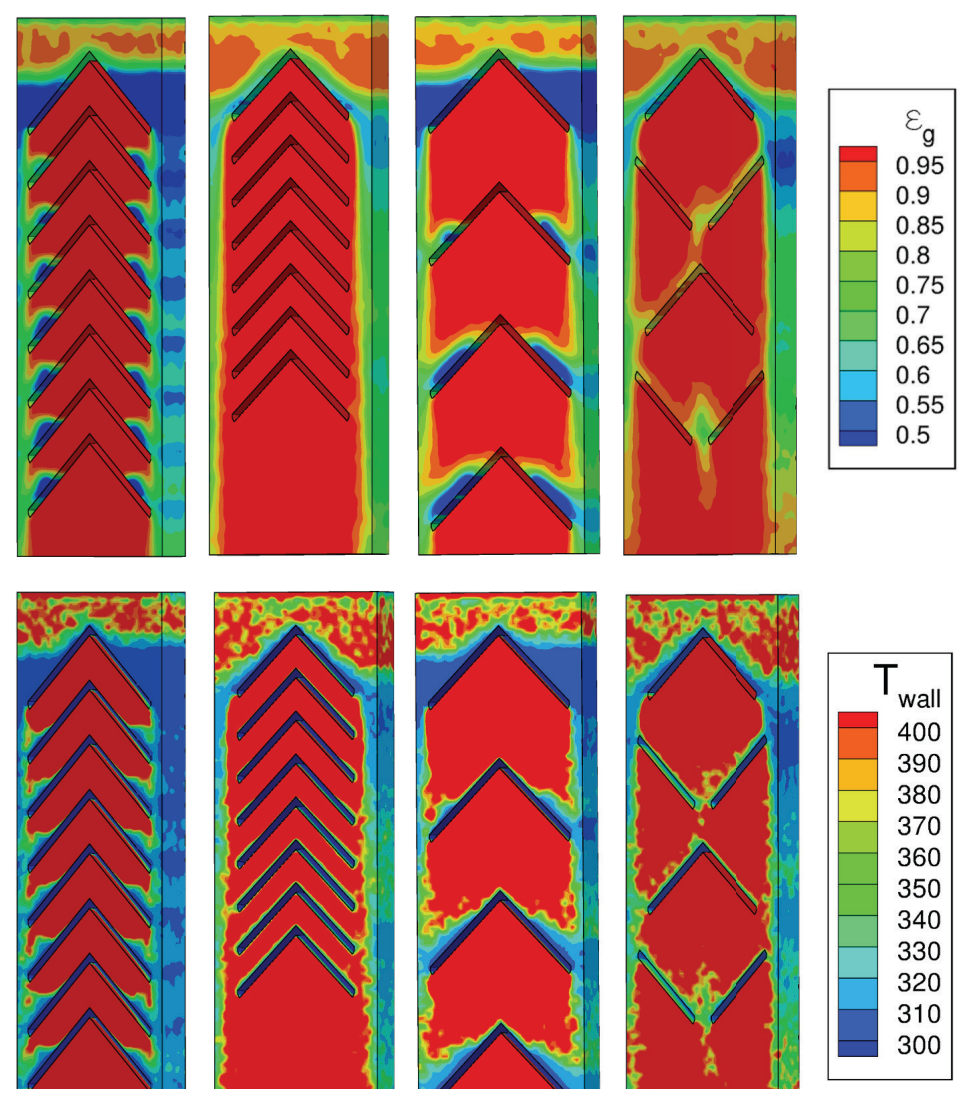

Figure 3. Falling-particle heat exchangers: Gas fraction profiles (top) and wall temperature $[\mathrm{K}\rceil$ profiles (bottom) are aiven for the four confiaurations at steadv 


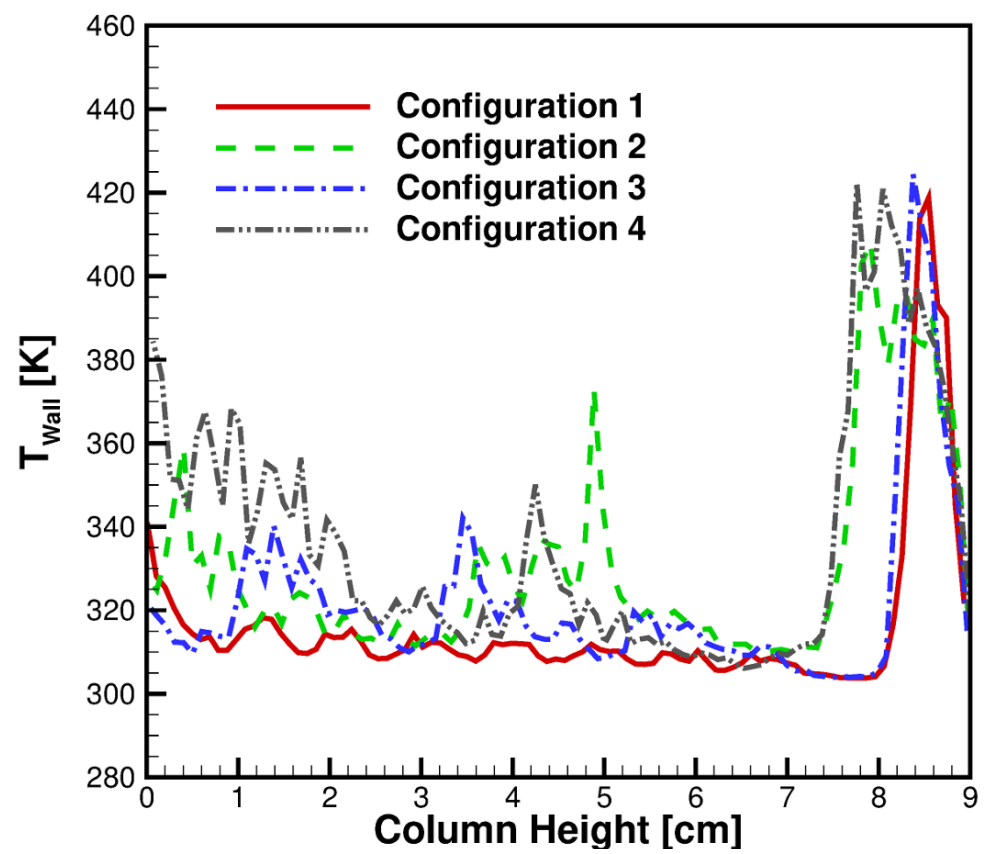

Figure 4. Heat Exchangers: Wall temperatures along the right side wall at steady state ( simulation time $=1 \mathrm{~s}$ ). Confiqurations are numbered from left to riaht in Fiqure

While simulations of the falling-particle heat exchangers show the expected qualitative behavior, a more quantitative assessment of the new BC is necessary. In Figure 5, the heat flux data for the first configuration (far left in Figure 3) is given in more detail. Specifically, the heat flux at the wall, averaged over the entire right-side wall, is tracked through the simulation and compared to the specified value, which is a linear ramp. The heat flux obtained using the new $\mathrm{BC}$ is in excellent agreement with the imposed value. Furthermore, the local heat flux associated with the gas phase and solid phase was extracted along the right side wall at steady state, and is plotted separately in Figure 6. The large fluctuation in solids heat flux near the inlet coincides with the spike in wall temperature (Fig. 4) and is due to reduced particle-wall contacts in a region of high void fractions (Fig. 3). Nonetheless, the simulated total wall flux is nearly constant along the column wall though the contribution from each phase varies spatially. A close inspection indicates that the spatial oscillations in the solids heat flux corresponds to baffle positions; these oscillations are out of phase with those of the gas phase, illustrating that the phases are indeed coupled and sum to the imposed value at any given location. 


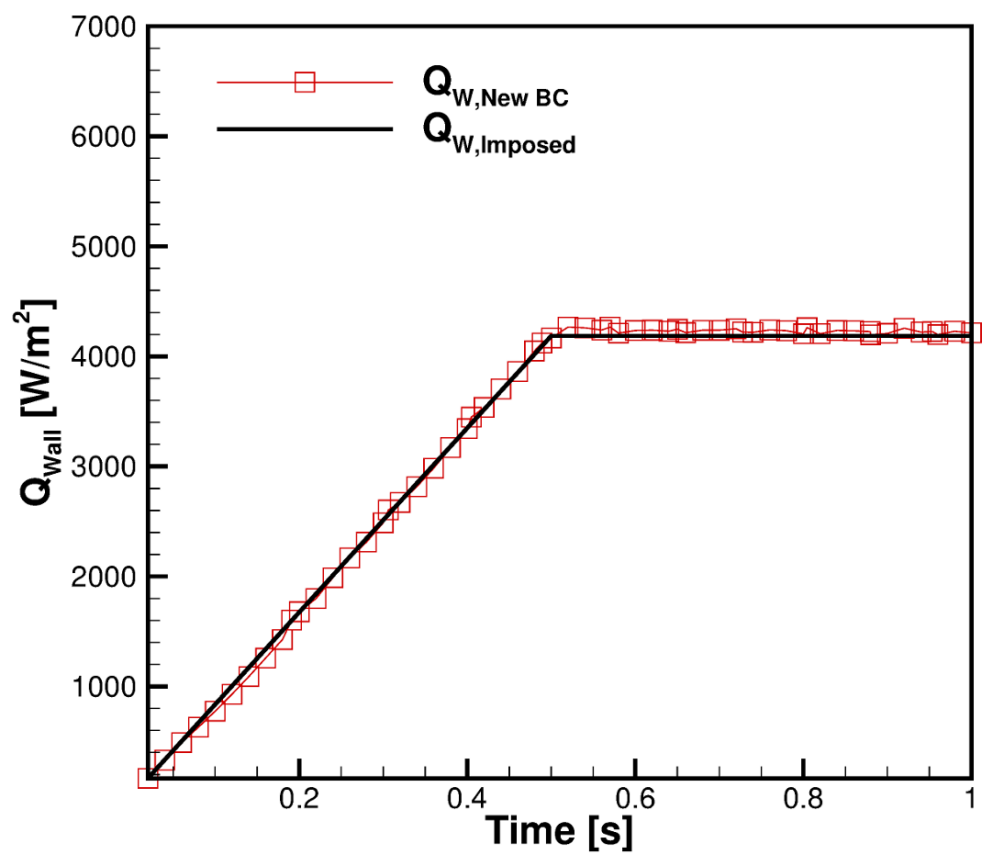

Figure 5. Heat Exchanger Configuration 1: Wall flux obtained using the new $B C$ is averaqed over the right wall and compared to the imposed wall flux as a function of

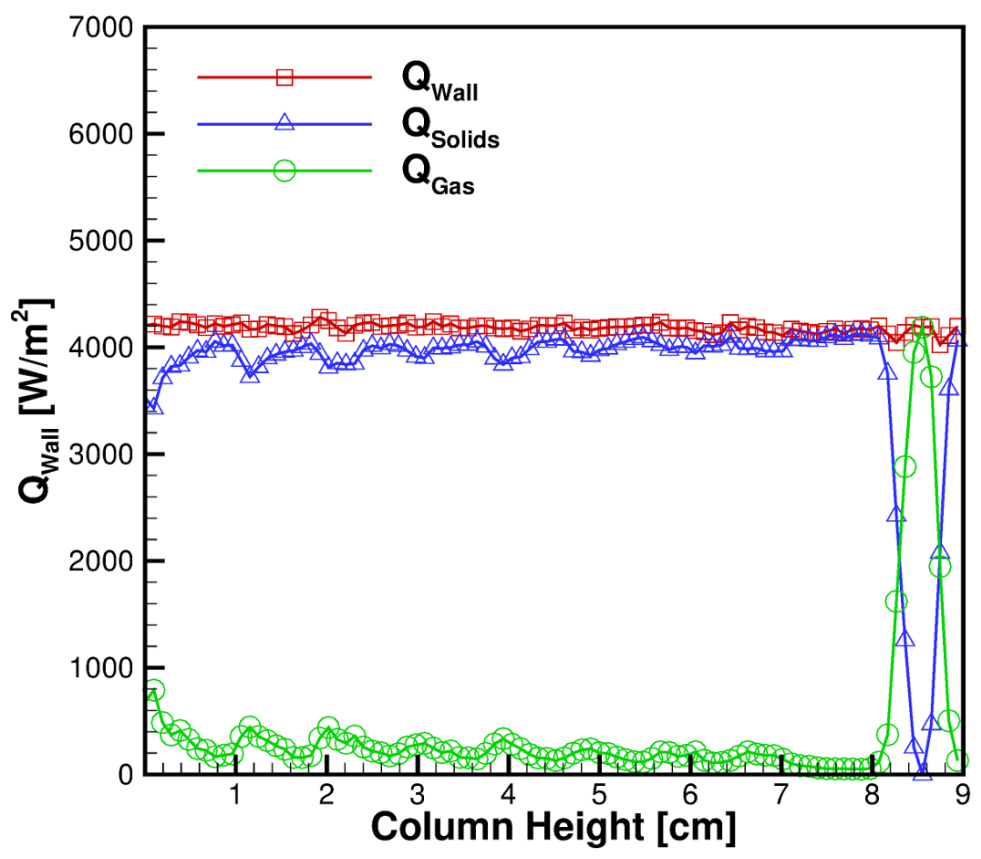

Figure 6. Heat Exchanger Configuration 1: Simulated heat flux data for the wall, solids, and aas alona the column height at steadv state (simulation time $=1 \mathrm{~s}$ ). 
The falling-particle heat exchangers are characterized by a stable steady state that makes the explicit approximation of "A" and "B" terms (Eq. 15) in the new BC sufficiently accurate. The bubbling bed, on the other hand, is characterized by bubbling instabilities, which lead to large temporal gradients in the solids heat flux. As a result, the simulation of the bubbling bed demonstrates the impact of hydrodynamic instabilities, and more specifically, large temporal gradients in the solids heat flux on the new BC. This effect is illustrated in Figure 7, where the simulated wall flux is averaged over the right side wall and compared to the imposed value for two different CFD time steps. The large, dynamic changes in solids heat transfer make the explicit prediction of the solids heat flux insufficiently accurate for the larger time step; the largest continuum time step $\left(10^{-4} \mathrm{~s}\right)$, which corresponds to reaching the desired residual $\left(10^{-5}\right.$ used in all simulations), yields a maximum error of $\sim 40 \%$ for the wall flux. However, the error can be made arbitrarily small by reducing $\Delta t$. For example, reducing the continuum time step by a factor of 2 reduces the maximum error to $\sim 1 \%$. As indicated by Figure 8 , the void fraction and temperature profiles for the bubbling bed again show the expected qualitative behavior. For areas of high solids concentration, the resulting wall temperatures are low.

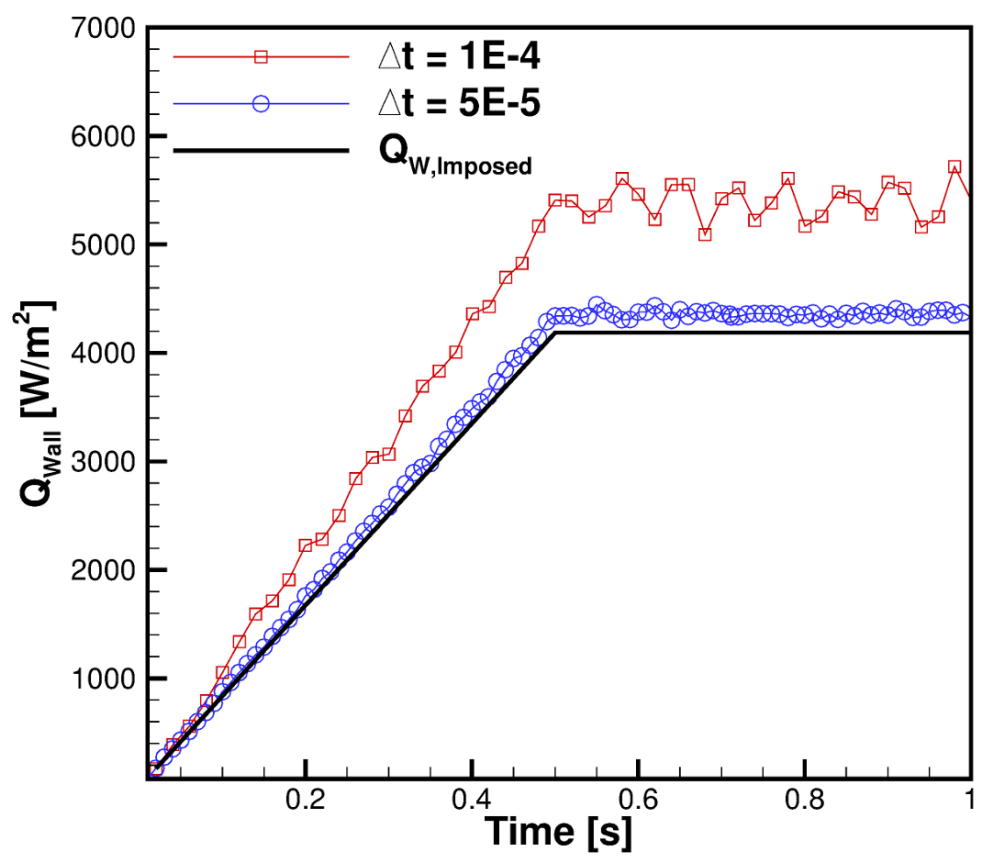

Figure 7. The simulated wall flux for two different continuum time steps is compared to the imposed wall flux as a function of time. 

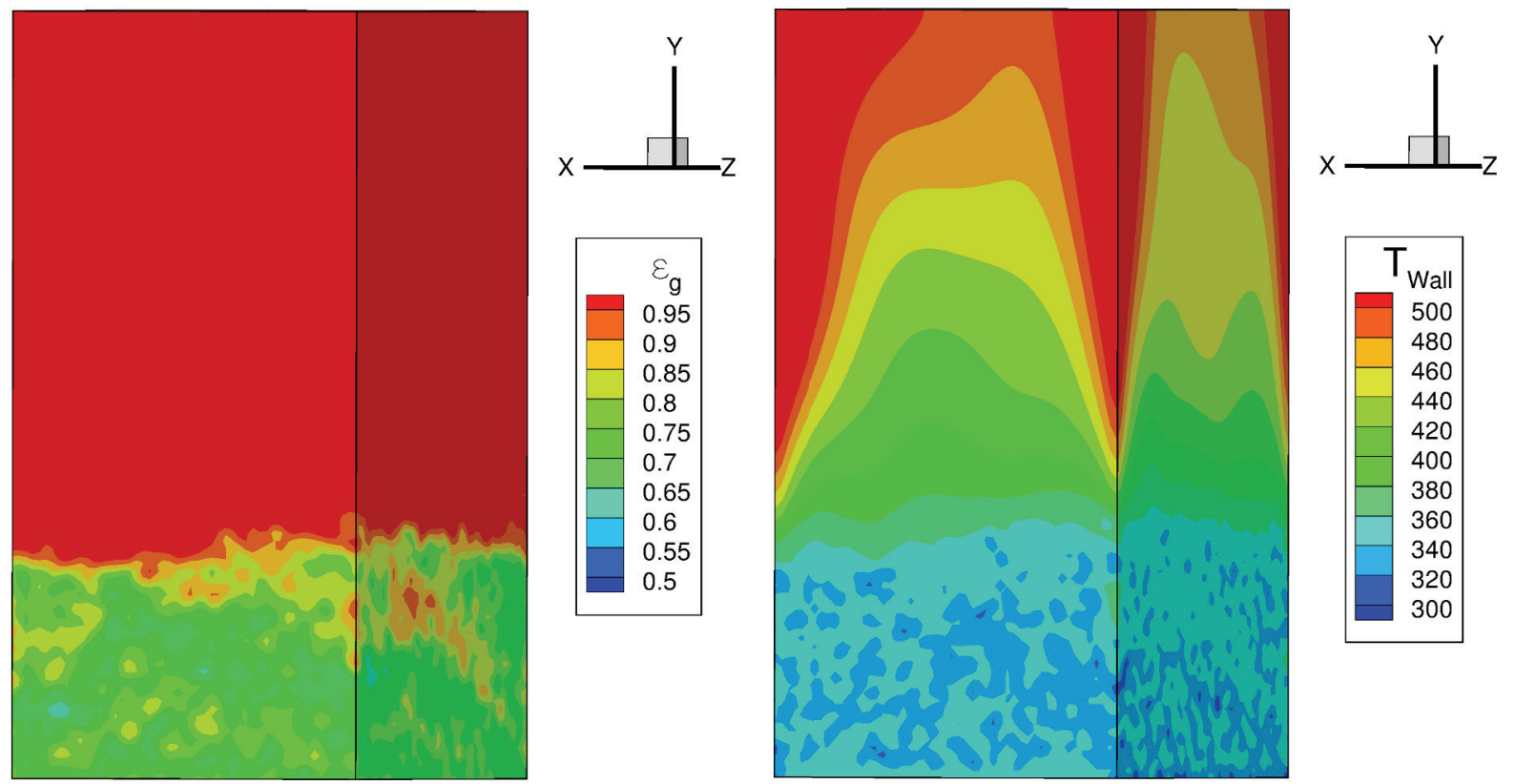

(a)

Figure 8. The hydrodynamic profile (a) and wall temperature profile (b) for the bubblina bed at a time of $1 \mathrm{~s}$.

\section{Concluding Remarks}

The current state-of-the-art for specifying a total heat flux to the boundary of multiphase flows requires the specification of a heat flux to each phase. By contrast, here we develop a boundary condition for total heat flux to the mixture, which allows the heat flux to each phase to vary in space and/or time. This new BC, targeted at CFD-DEM simulations, was utilized to simulate heat transfer between gas-solids flow and a heated wall in five systems: four fallingparticle heat exchangers with different baffle configurations, and one bubbling bed heat exchanger. For the falling-particle cases, which achieve a stable steady state, the simulated wall flux achieves a sufficient degree of accuracy using time steps dictated by the residual for the CFD solver. For the bubbling bed heat-exchanger, which is inherently unstable due to bubbling instabilities, the explicit coupling of CFD-DEM requires that the continuum time step be further reduced for sufficient accuracy.

The new boundary condition is of practical importance since many systems are more accurately described by a flux boundary condition rather than constant temperature. Such a boundary condition allows for wall temperature profiles in time and space to be determined and for potential "hot spots" to be identified. While only monodisperse particles with no internal temperature gradients and non-radiative mechanisms were explored for the CFD-DEM simulations considered in this work, the extension of the boundary condition to polydisperse CFD-DEM, particles with internal temperature gradients in CFD-DEM, radiative mechanisms, and/or the two-fluid models is possible. 


\section{Acknowledgements}

The authors are grateful for the funding provided by the Department of Energy under Grant DEEE0005954. This research used resources of the Oak Ridge Leadership Computing Facility located in the Oak Ridge National Laboratory, which is supported by the Office of Science of the Department of Energy under Contract DE-AC05-00OR2272.

\section{Literature Cited}

[1] Alazmi, B., Vafai, K., 2002. Constant wall heat flux boundary conditions in porous media under local thermal non-equilibrium conditions. International Journal of Heat and Mass Transfer 45, 3071-3087. doi:10.1016/S0017-9310(02)00044-3

[2] Amiri, A., Vafai, K., Kuzay, T.M., 1995. Effects of boundary conditions on non-darcian heat transfer through porous media and experimental comparisons. Numerical Heat Transfer, Part A: Applications 27, 651-664. doi:10.1080/10407789508913724

[3] Anderson, T.B., Jackson, R., 1967. Fluid mechanical description of fluidized beds. equations of motion. Ind. Eng. Chem. Fund. 6, 527-539. doi:10.1021/i160024a007

[4] Batchelor, G.K., O’Brien, R.W., 1977. Thermal or electrical conduction through a granular material. Proceedings of the Royal Society of London A: Mathematical, Physical and Engineering Sciences 355, 313-333. doi:10.1098/rspa.1977.0100

[5] Chaudhuri, B., Muzzio, F.J., Tomassone, M.S., 2006. Modeling of heat transfer in granular flow in rotating vessels. Chemical Engineering Science 61, 6348-6360. doi:10.1016/j.ces.2006.05.034

[6] Cong, T.N., He, Y., Chen, H., Ding, Y., Wen, D., 2007. Heat transfer of gas-solid two-phase mixtures flowing through a packed bed under constant wall heat flux conditions. Chemical Engineering Journal 130, 1-10. doi:10.1016/j.cej.2006.11.006

[7] Cundall, P.A., Strack, O.D.L., 1979. A discrete numerical model for granular assemblies. Géotechnique 29, 47-65. doi:10.1680/geot.1979.29.1.47

[8] Deen, N.G., Van Sint Annaland, M., Van der Hoef, M.A., Kuipers, J.A.M., 2007. Review of discrete particle modeling of fluidized beds. Chemical Engineering Science, Fluidized Bed Applications 62, 28-44. doi:10.1016/j.ces.2006.08.014

[9] Emady, H., Anderson, K., Borghard, B., Muzzio, F., Glasser, B., Cuitino, A., Prediction of Heating Time Scales of Particles in a Rotary Drum. AIChE Journal Submitted.

[10] Gidaspow, D., 1994. Multiphase Flow and Fluidization: Continuum and Kinetic Theory Descriptions. Academic Press.

[11] Gunn, D.J., 1978. Transfer of heat or mass to particles in fixed and fluidised beds. International Journal of Heat and Mass Transfer 21, 467-476. doi:10.1016/00179310(78)90080-7

[12] Kuipers, J. a. M., Prins, W., Van Swaaij, W.P.M., 1992. Numerical calculation of wall-tobed heat-transfer coefficients in gas-fluidized beds. AIChE J. 38, 1079-1091. doi:10.1002/aic.690380711

[13] Morris, A.B., Ma, Z., Pannala, S., Hrenya, C.M., 2016. Simulations of heat transfer to solid particles flowing through an array of heated tubes. Solar Energy 130, 101-115. doi:10.1016/j.solener.2016.01.033

[14] Morris, A.B., Pannala, S., Ma, Z., Hrenya, C.M., 2015. A conductive heat transfer model for particle flows over immersed surfaces. International Journal of Heat and Mass Transfer 89, 1277-1289. doi:10.1016/j.ijheatmasstransfer.2015.06.004

[15] Morris, A.B., Pannala, S., Ma, Z., Hrenya, C.M., 2016. Development of soft-sphere contact 
models for thermal heat conduction in granular flows. AIChE J. doi:10.1002/aic.15331

[16] Oschmann, T., Schiemann, M., Kruggel-Emden, H., 2016. Development and verification of a resolved 3D inner particle heat transfer model for the Discrete Element Method (DEM). Powder Technology 291, 392-407. doi:10.1016/j.powtec.2015.12.008

[17] Patil, A.V., Peters, E.A.J.F., Sutkar, V.S., Deen, N.G., Kuipers, J.A.M., 2015. A study of heat transfer in fluidized beds using an integrated DIA/PIV/IR technique. Chemical Engineering Journal 259, 90-106. doi:10.1016/j.cej.2014.07.107

[18] Patil, D.J., Smit, J., van Sint Annaland, M., Kuipers, J. a. M., 2006. Wall-to-bed heat transfer in gas-solid bubbling fluidized beds. AIChE J. 52, 58-74. doi:10.1002/aic.10590

[19] Pöschel, T., Schwager, T., 2005. Computational Granular Dynamics: Models and Algorithms. Springer Science \& Business Media.

[20] Ranz, W.E., Marshall, W.R., 1952. Evaporation from drops. Chem. Eng. Prog. 48, 141.

[21] Rong, D., Horio, M., DEM simulation of char combustion in a fluidized bed. Second International Conference on CFD in the Minerals and Process Industries CSIRO 65-70.

[22] Shi, D., Vargas, W.L., McCarthy, J.J., 2008. Heat transfer in rotary kilns with interstitial gases. Chemical Engineering Science 63, 4506-4516. doi:10.1016/j.ces.2008.06.006

[23] Spalding, D.B., 1980. Numerical computation of multi-phase fluid flow and heat transfer. Recent advances in numerical methods in fluids 1 139-167.

[24] Sreekanth, P., 2010. Computational Gas-Solids Flows and Reacting Systems: Theory, Methods and Practice: Theory, Methods and Practice. IGI Global.

[25] Stevens, A.B., Hrenya, C.M., 2005. Comparison of soft-sphere models to measurements of collision properties during normal impacts. Powder Technology 154, 99-109. doi:10.1016/j.powtec.2005.04.033

[26] Syamlal, M., 1998. MFIX documentation: Numerical technique. National Energy Technology Laboratory, Department of Energy, Technical Note No DOE/MC313465824.

[27] Syamlal, M., Gidaspow, D., 1985. Hydrodynamics of fluidization - prediction of wall to bed heat-transfer coefficients. AIChE Journal 31, 127-135.

[28] Syamlal, M., William, R., O’Brien, T., 1993. MFIX documentation: Theory guide. National Energy Technology Laboratory, Department of Energy, Technical Note DOE/METC95/1013 and NTIS/DE95000031.

[29] Vargas, W.L., McCarthy, J.J., 2002. Conductivity of granular media with stagnant interstitial fluids via thermal particle dynamics simulation. International Journal of Heat and Mass Transfer 45, 4847-4856. doi:10.1016/S0017-9310(02)00175-8

[30] Vargas, W.L., McCarthy, J.J., 2001. Heat conduction in granular materials. AIChE J. 47, 1052-1059. doi:10.1002/aic.690470511

[31] Zhou, Z.Y., Yu, A.B., Zulli, P., 2009. Particle scale study of heat transfer in packed and bubbling fluidized beds. AIChE J. 55, 868-884. doi:10.1002/aic.11823 